\title{
Evaluation and Severity Classification of Facial Paralysis using Salient Point Selection Algorithm
}

\author{
K. Anguraj \\ Assistant Professor, ECE \\ Sona College of Technology, \\ Salem, Tamilnadu, India
}

\author{
S. Padma, PhD \\ Professor, EEE \\ Sona College of Technology, \\ Salem, Tamilnadu, India
}

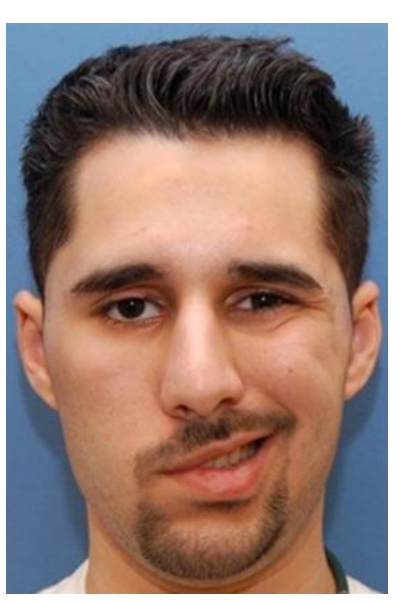

\begin{abstract}
Facial paralysis is a disease that affects the movement of one side of the face. This cause the disfigurement of face and difficulties in activities like eating, drinking, expressing and conversing. Reliable, quantitative and objective methods of evaluating the facial paralysis will be a precious tool for physicians to treat the patient with present condition. In this paper, a technique is proposed to evaluate the degree of facial paralysis. The proposed technique includes two phases namely training phase and testing phase. In training phase, a patient are requested to do four different facial expressions like closing of eyes, rising of eyebrows, opening of mouth and screwing of nose, and distance between salient points are calculated from each expression separately by using Salient Point Selection Algorithm(SPSA) and degree of facial paralysis is evaluated. During testing phase, the severity of facial paralysis is classified by well known neural network called Feed Forward Back Propagation Neural Network (FFBPN).
\end{abstract}

\section{Keywords}

Facial paralysis, Feed Forward Back Propagation Neural Network (FFBPN), salient point, Salient Point Selection Algorithm (SPSA), Degree Evaluation (DE).

\section{INTRODUCTION}

Facial paralysis is devastating disease that results when there is any damage to the facial nerve. The peripheral nervous system consists of spinal and cranial nerves. There are thirty two spinal nerves originate from spinal cord and traverses to brain and other parts of the body. These nerves carry the impulse between the brain and other parts of the body. The cranial nerves emerge from brain and brain stem. There are twelve pairs of cranial nerves and these are associated with head and neck. The facial nerve is the seventh cranial nerves and main function of this nerve is to move the muscles in the face [6]. This nerve is also responsible for secretion of saliva, tears, taste and sensation around ears. The other causes of facial paralysis are Ramsay - Hunt syndrome, Guillian Barre Syndrome, Melkerrson Rosenthal Syndrome, head or neck tumour, trauma and chronic middle ear function [6,9]. The symptoms of facial paralysis are deformity of face at rest and posed expressions, drooping of eyes, drooping of mouth, loss of taste, abnormal secretion of tears, and lumbness in the limbs.

The facial nerve paralysis is divided into central facial paralysis and peripheral facial paralysis[7,8].The central facial paralysis spares the forehead and eyes but affects all the lower facial muscles. The peripheral facial paralysis affects all voluntary and involuntary movements of facial muscles. The figure 1 shows the facial paralyzed image.
Fig 1: Paralyzed face

There are two different approaches for estimating the degree of facial paralysis. They are traditional approach and computer based approach. In traditional approach, the physician use subjective assessment for evaluation of facial paralysis. This approach comprises of marking of significant points on the face and assessed by calculating the distance between points. House categorized traditional grading system as gross, regional and specific. The gross category will assess overall facial function and provides the severity level of paralysis. Examples of gross grading system are HouseBrackman scale, May scale, and Pietersen scale. In regional category, assessment is made in terms of score for different areas of facial function separately. Smith scale, Yanagihara grading system, Sunnybrook facial grading system and Detailed Evaluation of Facial Symmetry are the examples of regional grading system. Specific grading system comprises of questions and observer has to respond to the question. Nottingham system and Burres-Fisch are the examples of specific grading system [1]. Computer based approach will assess the facial paralysis objectively by employing the specialized equipment and image processing technique [3,5,10]. Maximum Static Response Array (MSRA), Automated Face Image Analysis (AFIA), and Glasgow Facial Palsy scale are the examples of computer based facial grading system [11].

The paper is organized as follows. In section 2, the Salient Points Selection Algorithm is presented. In section 3 classification of degree of facial paralysis. In section 4 concludes the paper. 


\section{SALIENT POINTS SELECTION ALGORITHM}

\subsection{Facial expressions}

In order to assess the facial paralysis the physicians request the patient to do the following expressions like at rest, wrinkle forehead, blink, closure of eyes, wrinkle of nose, opening of mouth, whistle, grin and depress lower lip. Above expressions varies depending upon the grading system and region were paralyzed.

In the proposed method, the patients are requested do the facial expressions like closure of eyes, rising of eyebrows, opening of mouth and screwing of nose. Figure 2 shows the different facial expressions.

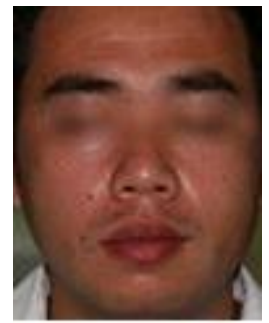

(a)

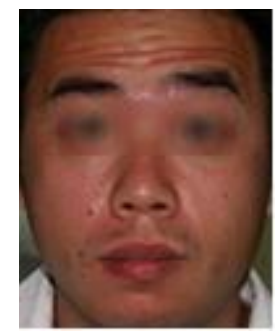

(c)

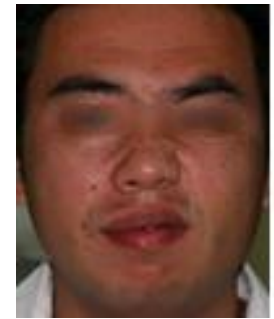

(b)

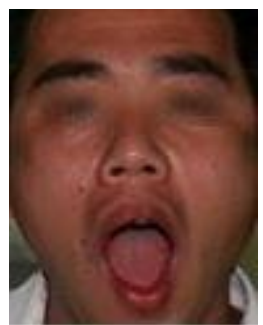

(d)
Fig 2: Different facial expressions (a) closing of eyes, (b) raising of eyebrows, (c) opening of mouth and (d) screwing of nose

\subsection{Salient Point Selection Algorithm}

\subsubsection{Salient points}

Salient points are most significant points are marked in the regions of facial features in different facial expressions $[2,13,14]$. Healthy persons can close the easily without any difficulties, but the facial paralyzed person can close the eyes with difficulties. The eyes will droops on the affected side of face. Patient was asked to close the eyes gently and salient points are marked on the topmost point teyl and bottommost point of the left eye beyl. And also on the right side the topmost point teyr and bottommost point beyr are marked. Figure 3 shows salient points during closing of eyes.

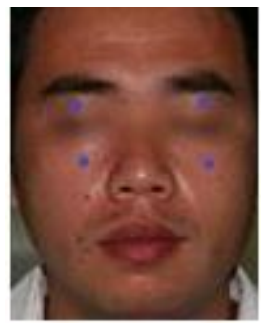

Fig 3: Salient points on facial expression 1
During the expression, rising of eyebrows the salient points are marked on the topmost tebl and bottommost bebl points on the left eyebrows. The salient points tebr and bebr are the topmost and bottommost points on the right eyebrows respectively. The figure 4 shows salient points on rising eyebrows.

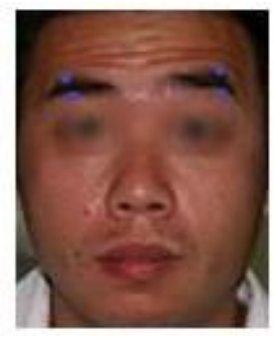

Fig 4: Salient points on facial expression 2

The salient points tml and bml are marked on the topmost and bottommost on left side of mouth respectively during the opening of mouth expressions. Similarly, tmr and bmr are the salient points the topmost and bottommost on the right side of mouth. Figure 5 shows salient points on expressions 3 .

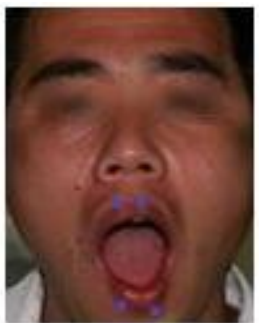

Fig 5: Salient points on facial expression 3

During the screwing of nose, the salient points are marked on the nose wing and both corner of the eyes. The salient points are marked on the centre of nose and corner of nose wing on the both sides. The salient points are also marked on the internal and external corner of both eyes. The salient points on the nose are cn, lnw and rnw, and the salient points on the corners of the left eye are inel and enel and on the corners of right eye are iner and ener. Figure 6 shows salient points on the expression 4 .

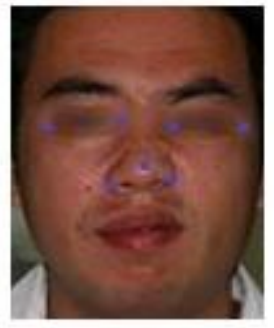

Fig 6: Salient points on facial expression 4

\subsubsection{Distance computations}

The distance between the salient points in every expressions are calculated. The distance between salient points on the left eye during closure of eyes are computed from the equation

$d(e y l)=\sqrt{(\text { teylx }- \text { beylx })^{2}+(\text { teyly }- \text { beyly })^{2}}$ 
where $\mathrm{x}$ and $\mathrm{y}$ are the coordinates of salient points on the left eye.

The distance between salient points right eye during closure of eyes are computed by using the equation

$d($ eyr $)=\sqrt{(\text { teyr } x-\text { beyl } x)^{2}+(\text { teyry }- \text { beyry })^{2}}$

where $\mathrm{x}$ and $\mathrm{y}$ are the coordinates of topmost and bottommost salient points on the right eye.

The distance are computed between the salient points on the left and right eyebrows by using the following equation

$d(e b l)=\sqrt{(t e b l x-b e b l x)^{2}+(t e b l y-b e b l y)^{2}}$

and

$d(e b r)=\sqrt{(\text { tebr } x-b e b r x)^{2}+(t e b r y-b e b r y)^{2}}$

During the screwing of nose, the distance is calculated between the center of nose and nose wing and also distance between the internal and external corners of eyes are obtained. The following equations are used to calculate the distance between salient points in those regions.

$d(n l w)=\sqrt{(\operatorname{cn} x-\ln w x)^{2}+(\operatorname{cn} y-\ln w y)^{2}}$

the above is to computed the distance between the center of the nose and left nose wing. Similarly, the distance between the centre and right nose wing is calculated by the using equation

$d(n r w)=\sqrt{(c n x-r n w x)^{2}+(c n y-r n w y)^{2}}$

In the same expression, the distance between the corners of left eye is obtained by using the equation

$d($ cle $)=\sqrt{(\text { inel } x-\text { enel } x)^{2}+(\text { inely }- \text { enely })^{2}}$

Similarly, the distance between the corners of right eye is obtained by using the following equation

$\mathrm{d}(\mathrm{cre})=\sqrt{(\text { iner } x-\text { ener } x)^{2}+(\text { inery }- \text { enery })^{2}}$

The salient point selection algorithm comprises of marking the salient points on the different regions with respect to different facial expressions and calculating the distance between salient points.

\subsubsection{Degree evaluation}

The degree of facial paralysis is evaluated by taking the ratios of distance between the salient points on affected side to distance between salient points on unaffected side. Degree evaluation is given as

$$
\begin{array}{ll}
\mathrm{DE}=\mathrm{DAF} / \mathrm{DUAF} & \text { if } \mathrm{DUAF}>\mathrm{DAF} \\
\mathrm{DE}=\mathrm{DUAF} / \mathrm{DAF} & \text { if } \mathrm{DUAF}<\mathrm{DAF}
\end{array}
$$

where DAF is the calculated distance between salient points on affected and DUAF is the calculated distance between salient on unaffected side. For example degree evaluation of an expression is obtained by the following expression
$\mathrm{DE}=\mathrm{d}($ eyl $) / \mathrm{d}($ eyr $)$

The overall facial paralysis degree of a patient is obtained by sum of all expressions degree to number of expressions.

$\mathrm{FDE}=$ sum of all degree of facial paralysis/ number of expressions.

For severity classification of facial paralysis the degree values are given as input the neural network.

\section{TESTING PHASE}

During testing phase, severity of facial degree is classified based on the overall degree of facial paralysis. In testing phase before classification of severity, preprocessing of images is carried out. Preprocessing includes image conversions, filtering and image enhancement. Preprocessing is followed by determining of regions, Salient Point Selection Algorithm and severity classification.

\subsection{Preprocessing of images}

A patient's images with different facial expressions are converted into gray scale images. Images with noises are removed by applying any one of filtering process. Weiner filter is used to remove noises from images with different facial expressions.

\subsection{Preprocessing of images}

The various facial features like eyes, eyebrows, nose and mouth are extracted from the different facial expression images $[4,12]$. The eyes are extracted from the closing of eye expression. The eyebrows are extracted from the rising of eyebrows expression. The mouth and nose are extracted from opening of mouth and screwing of nose expressions respectively. From each facial expression, the various salient points and distance between the salient points are calculated by SPSA algorithm. Apart from salient points marked on each expression few more neighbourhood points are marked near to salient point ie., the top and bottom of facial features.

The same SPSA algorithm can be used to calculate the distance between them. From the each expression, the maximum distances and minimum distances are calculated. The difference between the maximum distance on left side and maximum distance on right side is obtained. Similarly, difference between minimum distance on left side and minimum distance on right side is acquired. The above difference gives the level of paralysis on affected side to unaffected side of the face. The ratio between the maximum distances of affected side and maximum distances of unaffected side gives the degree of facial paralysis in that regions. Similarly, the ratios are obtained between minimum distances and minimum distances of salient points of both sides of face. Final degree evaluate values are given by the following expressions

$$
\begin{aligned}
& F D E_{n \text { min }}=\sum_{e=1}^{E}\left(D E_{\text {min }}^{e} / E\right) \\
& F D E_{n \text { max }}=\sum_{e=1}^{E}\left(D E_{\text {max }}^{e} / E\right)
\end{aligned}
$$

where $\mathrm{FDE}_{\mathrm{n} \min }, \mathrm{FDE}_{\mathrm{n} \max }$ are the final degree evaluated values, $\mathrm{DE}$ is degree of facial paralysis in each expression of a patient, and $\mathrm{E}$ is number of expressions. 


\subsection{Preprocessing of images}

The severity levels are categorized based on the final degree values. The classifications of levels are carried out by Feed Forward BackPropagation Neural Network (FFBPNN) [15]. The final degree evaluated values $\mathrm{FDE}_{\mathrm{n} \min }, \mathrm{FDE}_{\mathrm{n} \max }$ are given as the inputs to FFBPNN. Feed forward neural network consists of input layer, output layer and hidden layer.

The function of neural network is illustrated as

Steps 1: feed forward phase

Steps 2: backpropagation of error

Steps 3: updation of weights and bias.

The input from each unit of input layer receives the signal $\mathrm{FDE}_{\mathrm{n} \min }, \mathrm{FDE}_{\mathrm{n} \max }$ and given as the input to hidden layer. Each unit in hidden sums the weighted inputs, the output of hidden layer is obtained the by posing these inputs on to the activation functions. The outputs of hidden layer are given as input to the output layer. The error is computed from output from output layer and the target output. The error is propagated to hidden layer; the weight and bias of hidden layer and output layer are updated such that it produces the expected output.

\section{RESULT AND DISCUSSIONS}

The evaluation of degree of facial paralysis and severity classification system is realized by using MATLAB version 7.12.0(R2011a) and eight images of facial paralysis has been evaluated. The degree of facial paralysis is evaluated for each expression of a patient by using the SPSA algorithm and final degree of paralysis is evaluated by considering degree values from all the expressions. The final degree values are used as inputs to the Neural Network to classify the severity level of the disease. The figure 7 shows the grayscale images of all expressions. During the testing phase, the images are preprocessed to reduce the noise as well as to enhance the image. The Weiner filter is used to reduce the noise and it is shown in figure 8 . The extraction of various facial features is shown in Figure 9. The degree values of facial paralysis are calculated by using the SPSA algorithm is shown in table 1 . The severity of classification is shown in table 2 . The performance analysis of proposed technique for severity of facial paralysis classification is shown in table 3 .

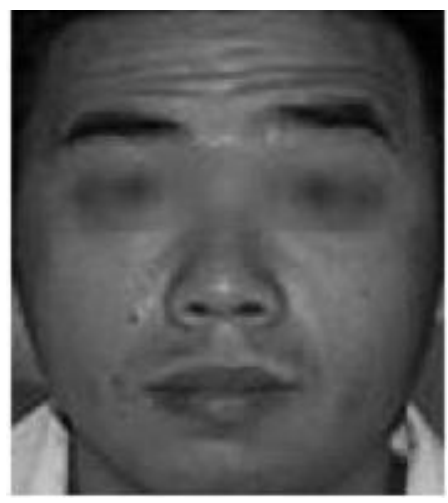

(a)

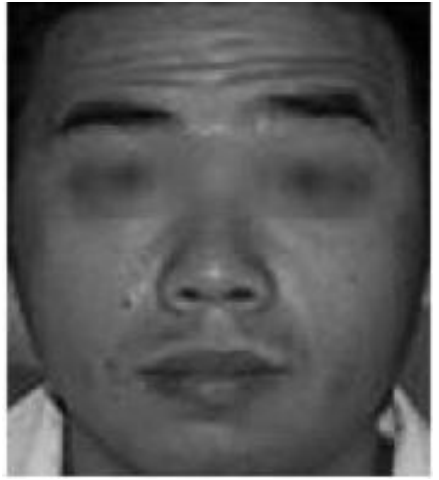

(b)

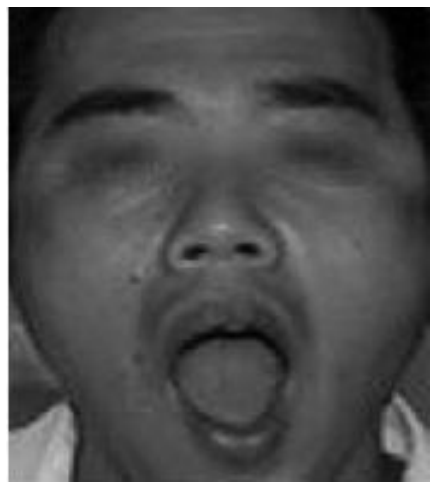

(c)

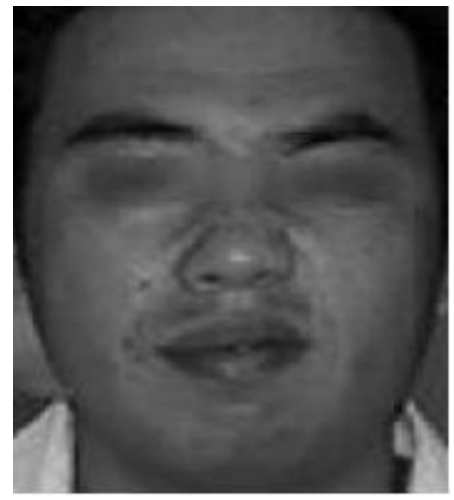

(d)

Fig 7: Gray scale images of different expressions (a) closing of eyes, (b) raising of eyebrows, (c) opening of mouth and (d) screwing of nose

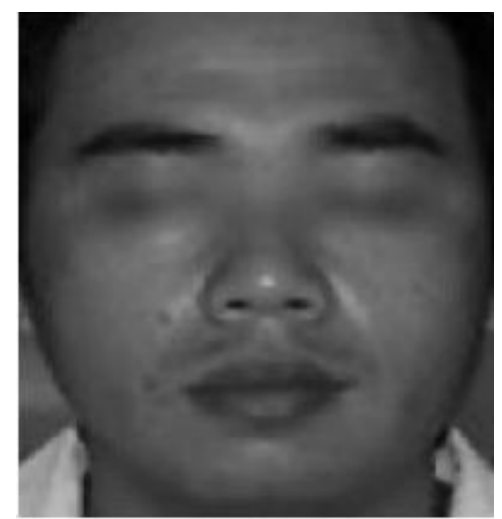

(a) 


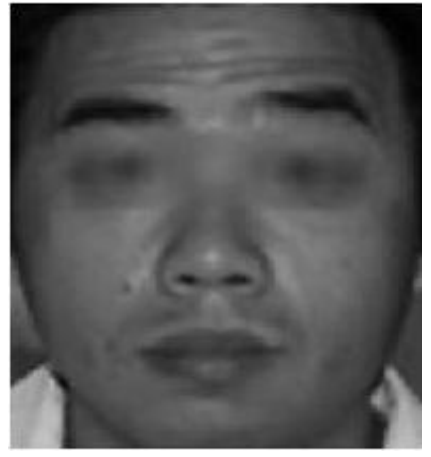

(b)

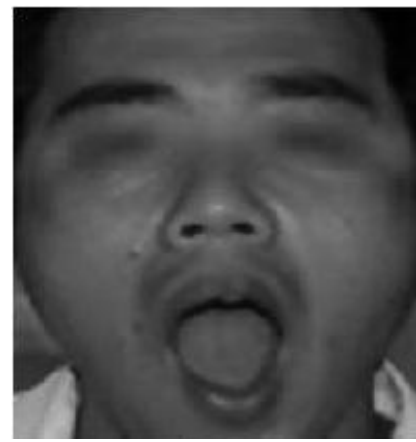

(c)

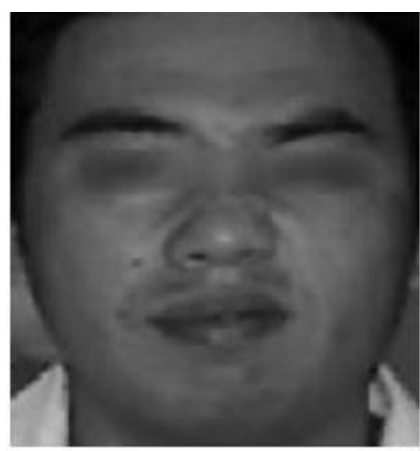

(d)

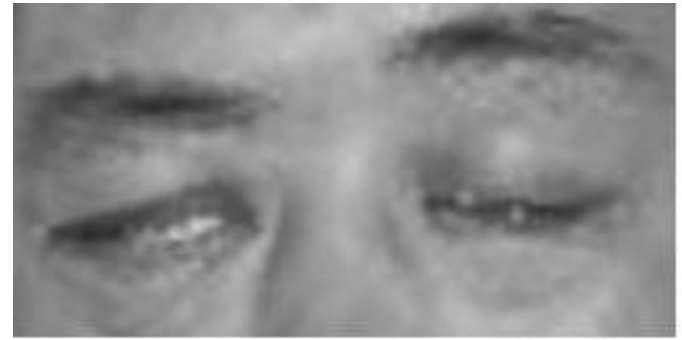

(a)

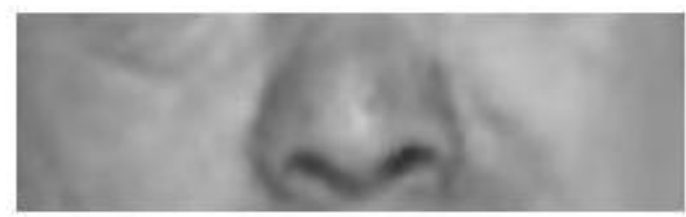

(b)

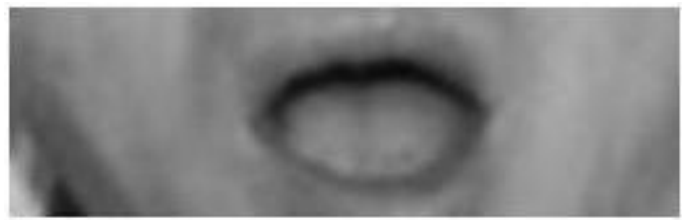

(c)

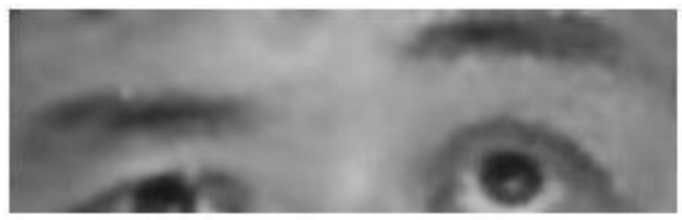

(d)

Fig 9: Extraction of various facial regions - (a) eyes, (b) nose, (c) mouth and (d) eyebrows

Fig 8: Preprocessing of images of expressions (a) closing of eyes, (b) raising of eyebrows, (c) opening of mouth and (d) screwing of nose

Table 1. Computed degree values

\begin{tabular}{|c|c|c|c|c|c|c|c|c|c|c|}
\hline \multirow{2}{*}{ Images } & \multicolumn{2}{|c|}{ Expression 1 } & \multicolumn{2}{c|}{ Expression 2 } & \multicolumn{2}{c|}{ Expression 3 } & \multicolumn{2}{c|}{ Expression 4 } & \multicolumn{2}{c|}{$\begin{array}{c}\text { Degree Values } \\
\text { (DE) }\end{array}$} \\
\cline { 2 - 13 } & Min & Max & Min & Max & Min & Max & Min & Max & Min & Max \\
\hline 1 & 18.39 & 15.56 & 56.15 & 56.56 & 11.31 & 12.72 & 2.83 & 12.72 & 22.17 & 35 \\
\hline 2 & 36.77 & 41.01 & 82.02 & 99 & 16.97 & 21.21 & 4.24 & 56.56 & 35 & 41.36 \\
\hline 3 & 4.24 & 4.25 & 39.6 & 91.92 & 8.38 & 14.14 & 16.26 & 51 & 17.12 & 40.32 \\
\hline 4 & 14.14 & 55.15 & 80.61 & 87.68 & 8.48 & 12.79 & 2.82 & 59.39 & 26.51 & 53.75 \\
\hline 5 & 2.82 & 12.72 & 12.72 & 29.69 & 14.14 & 43.84 & 25.45 & 29.69 & 13.78 & 28.98 \\
\hline 6 & 42.43 & 49.5 & 62.43 & 77.98 & 8.49 & 12.73 & 82.63 & 88.38 & 49 & 57.17 \\
\hline 7 & 80.61 & 86.27 & 83.44 & 87.68 & 45.25 & 56.57 & 41.01 & 49.5 & 62.58 & 70 \\
\hline 8 & 72.12 & 73.34 & 61.92 & 74.95 & 15.56 & 18.38 & 32.53 & 43.84 & 45.53 & 52.62 \\
\hline 9 & 50.57 & 62.88 & 71.78 & 78.02 & 42.25 & 43.08 & 21.28 & 24.87 & 48.93 & 49.76 \\
\hline
\end{tabular}


Table 2. Severity classification

\begin{tabular}{|c|c|}
\hline Image & Severity \\
\hline 1 & Moderate \\
\hline 2 & Mild \\
\hline 3 & Severe \\
\hline 4 & Severe \\
\hline 5 & Severe \\
\hline 6 & Normal \\
\hline 7 & Mild \\
\hline 8 & Normal \\
\hline 9 & Normal \\
\hline
\end{tabular}

Table 3. Performance analysis of proposed technique

\begin{tabular}{|c|c|c|c|c|}
\hline $\begin{array}{c}\text { Sensitivity } \\
(\boldsymbol{\%})\end{array}$ & $\begin{array}{c}\text { Specificity } \\
(\boldsymbol{\%})\end{array}$ & $\begin{array}{c}\text { Accuracy } \\
(\boldsymbol{\%})\end{array}$ & $\begin{array}{c}\text { Positive Predictive } \\
\text { Value (\%) }\end{array}$ & $\begin{array}{c}\text { Negative Predictive } \\
\text { Value (\%) }\end{array}$ \\
\hline 90 & 95 & 94 & 97 & 82 \\
\hline
\end{tabular}

\section{CONCLUSION}

In this paper, we proposed a technique that categories the severity of facial paralysis. The degree of paralysis is obtained by taking the difference between the affected side and unaffected sides of the face. The evaluation of degree of facial paralysis and severity classification are carried out by using SPSA algorithm and FFBPNN. The proposed methodology yielded the accuracy in evaluating diseases as well as categorization. In future works, we wish to improve the accuracy as well as to include few more severity level.

\section{REFERENCES}

[1] Nishida, Yen-Wei Chen, Matsushiro and Chihara, 2010,"An image based quantitative evaluation method for Facial Paralysis", Proceedings of the International Conference on Software Engineering and Data Mining (SEDM), pp. 706-709.

[2] Junyu Dong, Lijing Ma, Li-an Liu, Yang Lin, 2008,, “An approach for quantitative evaluation of the degree of facial paralysis based on salient point detection", Proceedings of IEEE International Symposium on the Intelligent Information Technology Applications Workshops, pp. 483-486.

[3] Shu He, Soraghan, O'Reilly and Dongshan Xing, 2009, "Quantitative Analysis of Facial Paralysis Using Local Binary Patterns in Biomedical Videos", IEEE Transactions on Biomedical Engineering, Vol. 56, No. 7, pp. 1864-1870.

[4] Lian Liu, Guanglin Cheng, Junyu Dong, Shengke Wang and Haibin Qu, 2010,"Evaluation of Facial Paralysis
Degree Based on Regions", Proceedings of the 3rd International Conference on Knowledge Discovery and Data Mining, pp. 514-517.

[5] Nevein M.M. Gharib, Sahar M. Adel and Nirmeen A. Kishk, 2011,"Reliability of three-dimensional motion analysis in assessment of Bell's palsy", Journal of American Science, Vol. 7, No. 9, pp. 126-134.

[6] Jessie M Vanswearingen, Jennifer S Brach, 1996, "The Facial Disability Index: reliability and validity of a disability assessment instrument for disorders of the facial neuromuscular system", Physical Therapy- Journal of the American Physical Therapy Association, Vol. 76, No.12, pp. 1288-1298.

[7] Jessie M Vanswearingen, 2008, "Facial Rehabilitation: A Neuromuscular Reeducation, Patient Centered Approach", Facial Plastic Surgery, Vol. 24, No.2, pp 250-259.

[8] S.He,J.J. Soraghan, and B.F.O'Reilly, ,2007 “Biomedical image sequence analysis with application to automatic quantitative assessment of facial paralysis", EURASIP Journal on Image and Video Processing, Vol.2007, No.4, pp. 812821-8128211.

[9] M.Kanerva, 2008 "Peripheral facial palsy grading, etiology, and Melkersson Rosenthal syndrome", Department of Otorhinolaryng, University of Helsinki Finland. 
[10] Stewart McGrenary, B.F.O'Reilly, and J.J Soraghan, 2005, "Objective grading of facial paralysis using artificial intelligence analysis of video data", in the Proceedings of the 18th IEEE Symposium Computer Based Medical Systems(CBMS'05), pp.587-592.

[11] T.Tanaka, J.Nemoto, M.Ohta, andT.kunihiro, 2004, "Evaluation of facial paralysis by amount of feature points movements at facial expressions", Proceedings of the 26th Annual International Conference of the IEEE EMBS, pp.1-5.

[12] Elham Bagherian, Rahmita.Wirza.Rahmat and Nur Izura Udir, 2009, "Extract of facial point", International Journal of Computer Science and Network Security, Vol.9, No.1, pp. 49-53.
[13] Frank Y shih, Shouxian Cheng, and Chao Fa Chuang, 2008, "Extracting face and facial features from color images", International Journal of Pattern Recognition and Artificial Intelligence, Vol.22, No.3, pp.515-534.

[14] Yulia Gizatdinova and Veikko Surakka, 2006, "Feature based detection of facial landmarks from neutral and expressive facial images", IEEETransactions on Pattern Analysis and Machine Intelligence, Vol.28, No.1, pp.35139.

[15] Maciej Mazurowskia , A , Piotr Habasa, A, Jacek Zuradaa, M, Joseph Lob, Y, Jay Bakerb, A \& Georgia Tourassib, D 2008,'Training Neural Network Classifiers for Medical Decision Making: The Effects of Imbalanced Datasets on Classification Performance', Neural Networks, Elsevier, vol. 21, pp. 427-436. 